\title{
High-quality colon cleansing and multiple neoplasia detection with 1L NER1006 versus mid-volume options: Post hoc analysis of phase 3 clinical trials
}

\section{다 (1) $(2)$}

\author{
Authors \\ Institutions \\ 1 Investigative Clinical Research, Annapolis, Maryland, \\ United States \\ 2 Service de Gastroentérologie, Hôpital Avicenne, \\ Bobigny, France \\ 3 Medical Affairs, Norgine Ltd., Harefield, United Kingdom \\ 4 University Hospitals Leuven, Department of \\ Gastroenterology and Hepatology, TARGID, KU Leuven, \\ Belgium
}

Michael S. Epstein ${ }^{1}$, Robert Benamouzig ${ }^{2}$, Juha Halonen ${ }^{3}$, Raf Bisschops ${ }^{4}$

\section{ABSTRACT}

submitted 9.10.2019

accepted after revision 8.1 .2020

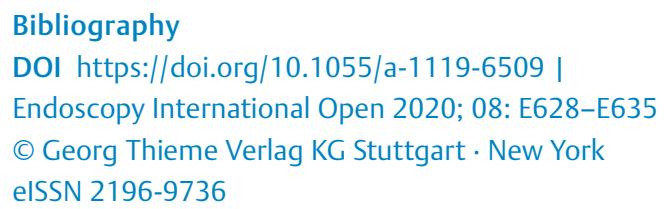

Corresponding author Juha Halonen, PhD, Medical Affairs, Norgine Ltd., Norgine House, Widewater Place, Moorhall Road, Harefield, Uxbridge UB9 6NS, UK

Fax: +441895825865

jhalonen@norgine.com

Supplementary material

Online content viewable at:

https://doi.org/10.1055/a-1119-6509
Background and study aims Multiple neoplasia increase the risk of colorectal cancer. High-quality cleansing may improve adenoma detection. We assessed whether a new bowel preparation can improve colon cleansing and multiple lesion detection.

Patients and methods This post hoc analysis of two randomized clinical trials in Europe and the US assessed the per study and combined cleansing efficacy of overnight split dosing with (preparation + clear fluids) $1+1 \mathrm{~L}$ polyethylene glycol (PEG) NER1006 versus 2+1L PEG + ascorbate (2LPEG) or $1+2 \mathrm{~L}$ oral sulfate solution (OSS) combined. Treatment-blinded central readers assessed cleansing quality using the Harefield Cleansing Scale (HCS). Patients with full segmental scoring were included. HCS segmental scores $0-4$ (high-quality $=\mathrm{HCS} 3-4$ ) were analyzed for NER1006 versus 2LPEG/OSS. Mean number of polyps or adenomas per patient (MPP/MAP) was calculated for treatments in patients with at least one polyp or adenoma.

Results In 1037 patients, NER1006 attained a greater rate of HCS 3 scores ( $29 \%$ vs. $20 \%$; $P<0.001)$ and HCS 4 scores ( $20 \%$ vs. $17 \% ; P=0.007$ ) versus 2 LPEG/OSS. More polyps (678 versus 585 ) and adenomas (397 versus 331 ) were detected with NER1006 $(N=517)$ versus 2 LPEG/OSS $(N=520)$. In all neoplasia-positive patients, with increasing minimal per-patient neoplasia multiplicity from 1 to 10 , NER1006 numerically improved MPP (difference \pm SE: $0.48 \pm 0.24$ to $3.89 \pm 3.37)$ and MAP $(0.47 \pm 0.26$ to $7.50 \pm 9.00)$ versus 2LPEG/OSS.

Conclusions Low-volume NER1006 enhances high-quality cleansing versus medium-volume 2LPEG or OSS, individually and when combined. NER1006 may consequently facilitate the detection of multiple neoplasia in patients.

\section{Introduction}

Colonoscopy for colorectal cancer (CRC) screening reduces CRC incidence and mortality [1-3] Adenoma detection rates (ADR) are associated with $C R C$ rates and mean numbers of polyps (MPP) and adenomas (MAP) per patient both correlate strongly with ADR [4-7].
To ensure that precancerous and cancerous lesions can be detected, full visualization of the colonic mucosa during colonoscopy is required. At a minimum, this requires adequate pre-procedural bowel preparation, ideally using an overnight split-dosing regimen $[8,9]$. While clinical guidelines do not yet address high-quality cleansing, recent data suggest that detec- 
tion of polyps, adenomas, and sessile or serrated polyps may increase further with high-quality colon cleansing [10-13].

Recent studies have improved our understanding of CRC risk versus detected adenoma size and multiplicity of lesions. Diminutive polyps $(<5 \mathrm{~mm}$ in size) alone may present an increased risk of advanced metachronous adenomas, compared with no polyps at all [14]. A recent study found an increased risk with three or more diminutive adenomas even versus one to two larger non-advanced adenomas [15]. When compared with larger adenomas, however, diminutive adenomas alone even with advanced morphology - may present a limited relative risk [16]. Importantly, that picture changes significantly if any small adenoma $(6-9 \mathrm{~mm})$ is also present; identification of a single diminutive adenoma in addition to an already detected small adenoma may significantly increase a patient's risk for advanced adenomas, particularly for patients undergoing colonoscopy for screening purposes $[17,18]$.

Adenoma multiplicity is a risk factor for fatal post-colonoscopy CRCs [19]. A registry study stratified more than 40,000 patients as either high-risk ( $\geq 5$ adenomas) or intermediate risk (3 to 4 small adenomas). High- and intermediate-risk patients had more advanced adenomas than patients with a single adenoma $\geq 10 \mathrm{~mm}$ ( $12.3 \%$ and $8.0 \%$ vs. $6.1 \%$ ) [20]. The only factor with an odds ratio $>2$ for advanced adenomas was adenoma multiplicity [21]. Another study in veterans showed a higher 10 -year incidence of advanced neoplasia in patients with baseline CRC or advanced adenomas than in patients with one to two adenomas or no neoplasia $(43.7 \%$ and $21.9 \%$ vs. $6.3 \%$ or $4.1 \%$ ) [22]. Interestingly, patients with three or more small adenomas carried a $66 \%$ risk of developing advanced neoplasia compared with patients with advanced adenomas (odds ratios 2.50 vs. 3.77). This is a dramatic increase from the $29 \%$ relative risk $(6.3 / 21.9)$ in patients with one to two adenomas. A microsimulation modeling of community-based health care data on ADR variation and cancer risk among 57,588 patients points in the same direction [23]. Detection and removal of small adenomas ( $<5 \mathrm{~mm}$ in size) had an independent risk-lowering effect for both cancer cases and cancer deaths, but the detection and removal of all adenomas of all sizes had the largest protective effect.

A re-analysis of eight tandem colonoscopy studies recently reported that diminutive adenomas are missed more frequently than larger adenomas [24]. Hence to date many patients, based on their colonoscopy findings, may have been assigned no or an inadequately long time for surveillance because of missed adenomas. If high-quality cleansing can be routinely bettered, and this enables detection of more polyps and adenomas, then logically patient protection against CRC could and should also be improved.

The Harefield Cleansing Scale (HCS) is a validated scale for bowel cleansing assessment.[25] Segmental scores range from 0-1 (failure) and 2 (successful) to 3-4 (high-quality). Successful bowel preparation means that the least clean segment has, at most, only residual opaque liquid or semi-solid stool left that is fully removable with appropriate suction (score 2). The HCS includes two high-quality segmental cleansing scores, achievable only if no washing is required: score 3 is stool-free but al- lows for clear liquid to be present, while score 4 requires an empty and clean colon segment. The term stool-free has been used here as a simple descriptor to quickly distinguish in plain language the high-quality HCS segmental cleansing scores 3-4 from the adequate quality HCS score 2.

NER1006 (PLENVU, a registered trademark of the Norgine group of companies) is the first $1 \mathrm{~L}$ polyethylene glycol (PEG) bowel preparation. It has been approved in 29 countries, including the United States and across Europe. To date, NER1006 is estimated to have been used in more than one million patients [26]. The low reconstituted volume of NER1006 is achieved by increasing the ascorbate components, relative to previous 2 L PEG and ascorbate (2LPEG), and delivering them in the second dose only. The efficacy and safety of NER1006 was established in a programme of three similarly designed Phase III multicenter, randomized, endoscopist- and central readerblinded, actively controlled clinical trials conducted in Europe and the United States, using three dosing regimens [27-29] The primary endpoint of the effect of cleansing quality on the HCS was assessed strictly by treatment-blinded central readers, a method that minimizes inter-reader assessment variability [30].

The current post hoc analysis included, for comparative reasons, only one dosing regimen (overnight split-dosing; widely adopted as the gold standard), which was assessed in two of the three trials. In the original trials, patients were randomly assigned in equal ratios to receive either NER1006 or 2LPEG (MORA study) or oral sulfate solution (OSS) (NOCT study). The MORA study included a NER1006 morning only regimen third arm, which was excluded from the current post hoc analysis. All treatments were administered per their approved labels: 2LPEG allowed for meals, including light dinner, on the day before colonoscopy, but OSS permitted only breakfast the day prior to the procedure. The NER1006 regimen permitted light breakfast and light lunch. Additional clear fluid intake as part of the treatment was mandatory for NER1006 and OSS and strongly recommended for 2LPEG. The amounts are specified in the Supplementary Table 1, however, the ingested volumes of additional clear fluids were not recorded.

The HCS high-quality cleansing efficacy of NER 1006 was assessed versus 2LPEG/OSS. The potential of NER1006 to facilitate detection of multiple lesions has not yet been reported, so a key analysis here was to also assess in detail the comparative detection of multiple neoplasia.

\section{Patients and methods}

\section{Patients}

Patients included in the MORA and NOCT trials were males and females aged 18 to 85 who required screening, surveillance, or diagnostic colonoscopy. Inclusion and exclusion criteria were near identical across the trials and have been described previously [27, 29].

Patients were treated with overnight split-dosing regimens of either NER1006, 2LPEG (MOVIPREP, Norgine Ltd, Harefield, United Kingdom; assessed in the MORA trial), or OSS (SUPREP, 
Braintree Laboratories, Braintree, Massachusetts, United States; assessed in the NOCT trial).

Cleansing efficacy was analyzed by treatment-blinded central readers using the HCS on colonoscopy videos by site endoscopists; the latter recorded polyp counts as per usual clinical practice. Adenoma counts were verified by laboratory analysis.

The predefined analysis set, the modified full analysis set (mFAS: full analysis set excluding any patient who failed laboratory screening after randomization and who also did not take any study treatment), used as the primary analysis population for the original study cleansing efficacy analyses, was included in this new post hoc analysis for clarity.

The main focus of this post hoc analysis is a subset of the mFAS, the mFAS2, from which patients with missing colonoscopy data imputed as failures were excluded. The mFAS2 reflects cleansing performance as seen by endoscopists in the clinic by only including patients who actually underwent a colonoscopy with bowel cleansing assessment.

\section{Study design}

This post hoc analysis used patient-level data from the overnight split-dosing arms of both the MORA and NOCT trials to analyze treatment effects on colonic HCS segmental cleansing scores and polyp and adenoma counts in the overall colon. Specifically, we focused on the attainment of high-quality cleansing and on the detection of multiple neoplasia per patient. We performed these analyses per trial and on data from both trials.

\section{Assessments}

The HCS was used for efficacy endpoint assessments and post hoc analyses (Supplementary Table2) [25,27,28]. Overall cleansing success and polyp/adenoma detection rates in mFAS have been reported elsewhere $[27,28]$. Four analyses were performed:

1. Attainment of high-quality cleansing. The proportion of high-quality cleansed colon segments (HCS 3-4) was assessed per treatment group in the original Phase III trial analysis set, mFAS. The analysis was performed by study. Because 2LPEG and OSS have between them shown a comparable cleansing efficacy in two recent randomized controlled trials, the NOCT and MORA data sets were combined for an analysis of combined NER1006 versus combined 2LPEG/OSS $[31,32]$. Hereafter, combined NER1006 and combined 2LPEG/OSS will simply be referred to as NER1006 and 2LPEG/ OSS, respectively. The HCS divides the colon into five segments, so the segmental sample size for all these analyses was five times the number of patients. A further analysis was performed in the mFAS2 population: distribution of HCS segmental scores 0-4 was analyzed for NER1006 versus 2LPEG/OSS to assess whether NER1006 predominantly improves the rate of stool-free cleansing with remaining clear fluid only (a shift from HCS 2 to HCS 3) or improves the rate of empty and clean segments (a shift from HCS 2 to HCS 4). mFAS2 data sets were used for subsequent analyses of polyp and adenoma detection.
2. MPP and MAP in all mFAS2 patients. MPP and MAP were analyzed separately for each study and for NER1006 versus 2LPEG/OSS.

3. MPP and MAP in mFAS2 patients with at least one neoplasia. MPP and MAP were analyzed separately in lesion-positive patients sequentially from those with at least one lesion up to those with at least ten lesions. This analysis aimed to assess the intra-patient lesion multiplicity detection potential of NER1006 versus 2LPEG/OSS. This analysis was not performed at the study level due to rapidly diminishing sample sizes with increasing polyp and adenoma counts.

4. Detection rates of mFAS2 patients with at least one neoplasia. This analysis assessed the comparative detection rates of patients with multiple polyps or, separately, multiple adenomas. This analysis aimed to assess the inter-patient lesion multiplicity detection potential of NER1006 versus 2LPEG/ OSS.

\section{Statistics}

All analyses were carried out using the statistical package $R$ v3.4.2 (The R Foundation, 2015). For each trial, patient- and segment-level data were extracted and central readers' HCS scores were documented according to treatment group. The proportion of high-quality segments per treatment group and the distribution of segmental scores per treatment group were calculated from documented segmental HCS scores. Cleansing success rates are reported as percentages. $P$ values comparing NER1006 with the comparator bowel preparation were estimated using the one-sided Student's t-test. Where a lower frequency of (stool-containing) segments is presented, the presented $P$ value is, for clarity, 1 -P generated by the 1 -sided $t$-test.

MPP and MAP results for patients with at least one neoplasia are presented as means + standard errors (SE). Rather than using standard deviations which show the possible variability of responses, the true mean values were of greater interest in the current analysis and the plotted SE also graphically captured the increased variability associated with decreasing sample sizes for patients with five or more neoplasia. Further statistical analysis was not deemed meaningful due to rapidly declining sample sizes in both groups as the minimum number of polyps or adenomas per patient increased. Patients with more than 10 neoplasia are more likely than patients with one to two neoplasia to be identified as high-risk patients and they would be assigned shorter surveillance intervals regardless of having occasional missed adenomas. The clinically most relevant multiplicity range to detect is probably from at least one to a maximum of ten polyps or adenomas per patient.

\section{Results}

\section{Patient characteristics and disposition}

Baseline characteristics for the full analysis sets in the MORA and NOCT trials have been reported previously [27,28]. A total of 1037 patients were included in this analysis; baseline demographics for this population are provided in Supplementary Table 3 and patient disposition is presented in Supplementary 
Fig. 1. The mFAS2/FAS ratios were comparable within each analyzed trial.

\section{Attainment of high-quality cleansing}

NER1006 achieved more high-quality cleansed segments (with HCS segmental score 3-4) than its comparators in the predefined analysis set mFAS. Specifically, NER1006 achieved more high-quality cleansed segments than 2LPEG (47.5\% [653/ $1375]$ vs. $30.2 \%[411 / 1360] ; P<0.001)$ or OSS (45.0\% [621/ $1380]$ vs. $40.2 \%$ [563/1400]; $P=0.005$ ) ( Fig. 1a). The combined trials analysis in mFAS patients also showed a significantly higher rate of high-quality cleansed segments for patients on NER1006 versus 2LPEG/OSS (46.2\% vs. 35.3\%; $P<0.001)$. Hence in patients with colonoscopy data assessed by treatment-blinded central readers, NER1006 was the only bowel preparation to attain $45 \%$ or more high-quality segments per treatment group, and it did so reproducibly in the primary analysis population of two randomized controlled trials.

In mFAS2 patients, the segmental score distributions showed higher rates of stool-free cleansing scores (HCS 3 ) and all empty and clean colon segments scores (HCS 4) with NER1006 versus 2LPEG/OSS (29.2\% vs. $20.1 \%$; $P<0.001$ and $20.0 \%$ vs. $17.4 \%$; $P=0.007$ ) ( Fig. 1 b). There was a corresponding decrease in stool-containing segments with HCS score 2 ( $48.5 \%$ vs. $59.3 \%$; $P<0.001)$. Hence the most frequent cleansing improvement with NER 1006 versus 2LPEG/OSS was the improved attainment of stool-free cleansing.

\section{MPP and MAP in all patients}

More polyps (678 versus 585) and adenomas (397 versus 331) were detected with NER1006 $(\mathrm{N}=517)$ versus 2LPEG/OSS $(\mathrm{N}=$ 520). This higher total detection with NER1006 was observed also within each of the trials. In MORA mFAS2 patients (262 versus 260), more polyps (350 versus 290) and more adenomas (159 versus 138) were detected with NER1006 versus 2LPEG. In NOCT mFAS2 patients (255 versus 260), more polyps (328 versus 295 ) and more adenomas (238 versus 193) were detected with NER1006 versus OSS.

Most patients had a maximum of 10 polyps or adenomas. Very few patients had more than 10 polyps (NER1006: five patients with $11,13,15,30$, and 37 polyps, respectively; and 2LPEG/OSS: three patients with 11, 12, and 14 polyps, respectively) or more than 10 adenomas (NER1006: one patient with 37 adenomas; and 2LPEG/OSS: two patients with 11 and 12 adenomas, respectively).

Patients in mFAS2 showed numerically higher MPP with NER1006 than with 2LPEG (mean \pm SE: $1.34 \pm 0.18$ vs. $1.12 \pm$ $0.11 ; P=0.143)$, OSS ( $1.29 \pm 0.19$ vs. $1.13 \pm 0.11 ; P=0.239)$, or the 2 LPEG/OSS $(1.31 \pm 0.08$ vs. $1.13 \pm 0.08 ; P=0.105)$ ( $>$ Fig. 2 ). Similarly, patients in mFAS2 showed numerically higher MAP with NER1006 than with 2LPEG $(0.62 \pm 0.09$ vs. $0.55 \pm 0.08$; $P=$ $0.287)$, OSS $(0.95 \pm 0.18$ vs. $0.75 \pm 0.09 ; P=0.152)$, or the 2LPEG/OSS ( $0.78 \pm 0.10$ vs. $0.65 \pm 0.06 ; P=0.128)$.

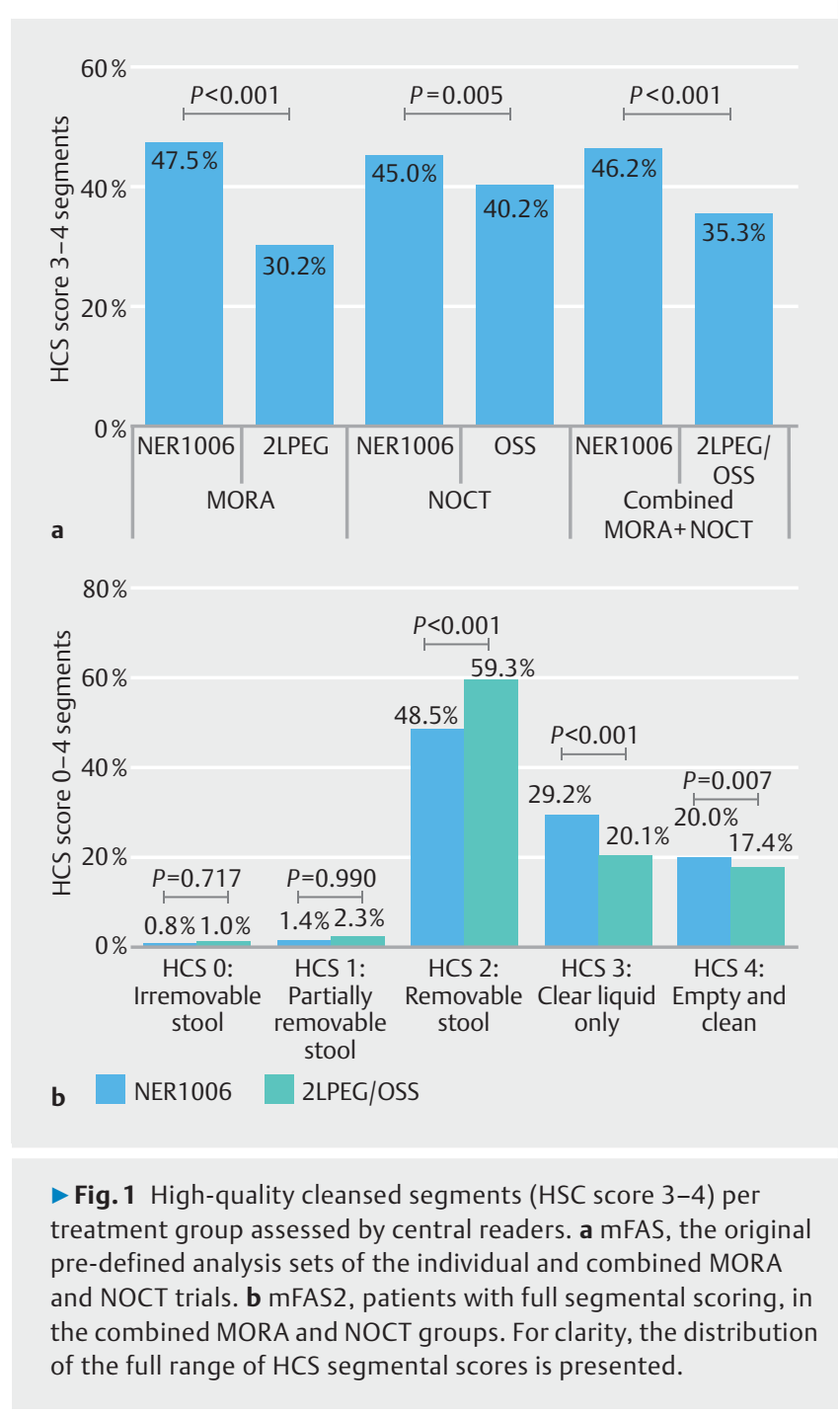

\section{MPP and MAP in patients with at least one neoplasia}

In the mFAS2 populations with at least one polyp per patient there was a consistent positive trend for numerically greater MPP with NER1006 versus 2LPEG/OSS in patients with at least one polyps per patient (difference \pm SE: $0.48 \pm 0.24-3.89 \pm$ 3.37 polyps per patient) ( $\mathbf{F i g . 3 a )}$. Similarly, MAP was numerically greater with NER 1006 versus 2LPEG/OSS in all patients with at least 1 adenomas (difference \pm SE: $0.47 \pm 0.26-7.50 \pm$ 9.00 adenomas per patient) ( $\mathbf{F i g} \mathbf{3} \mathbf{3 b}$ ). Together these two results indicate a positive trend in favor of NER1006 for the identification of multiple neoplasia in patients.

\section{Detection rates of patients with at least one neoplasia}

In mFAS2 patients, as the number of detected polyps per patient increased, a positive and consistent trend (compatible with the trends seen for MPP and MAP) showed that in patients with three or more polyps, numerically more patients with multiple polyps were detected with NER1006 than 2LPEG/OSS ( Table 1). 


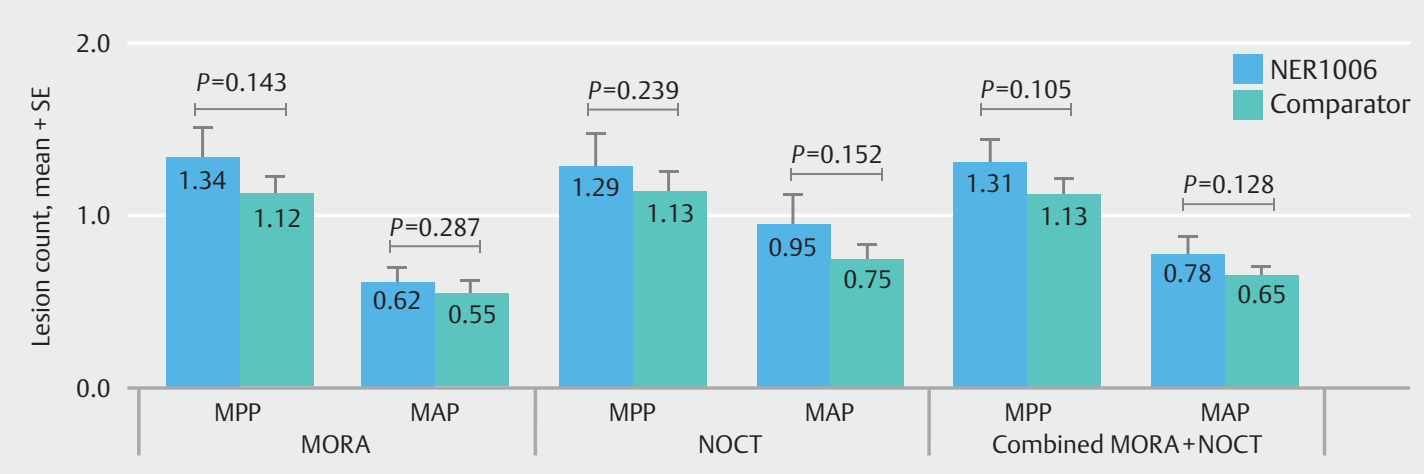

Fig. 2 Polyp and adenoma counts in mFAS2. Comparative attainment in mFAS2 of MPP and MAP per treatment and study, and at a combined trial level, in all patients.

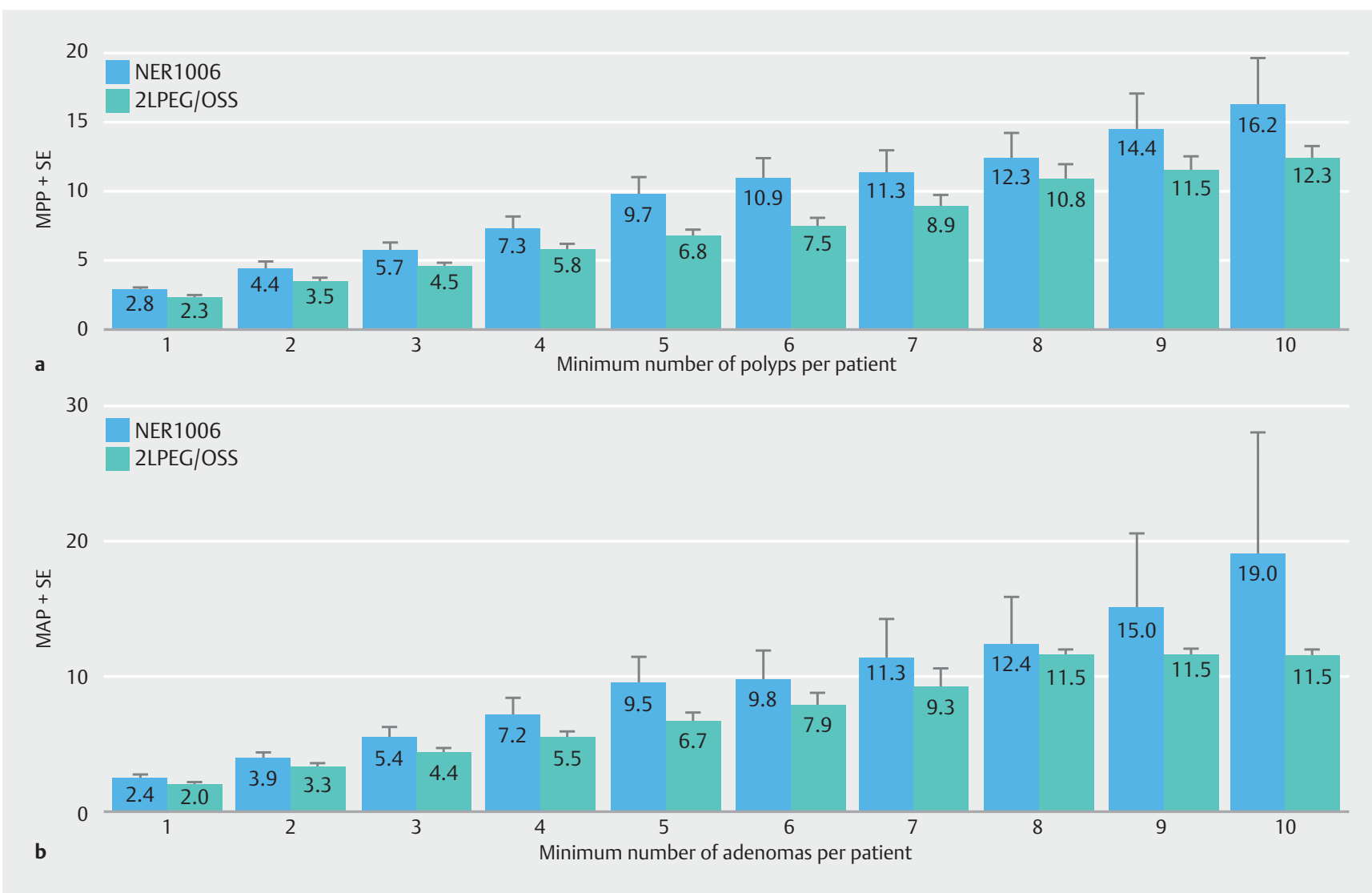

- Fig. 3 Comparative attainment in mFAS2 of MPP and MAP per treatment at a combined trial level. a Patients with at least one polyp. b Patients with at least one adenoma.

A similar consistent positive relationship was observed for patients with multiple adenomas. Patients with two or more adenomas were consistently numerically more often detected with NER1006 than with 2LPEG/OSS.

\section{Discussion}

We analyzed the relative colon-cleansing efficacy of the $1 \mathrm{~L}$ NER1006 versus two alternative bowel preparations, which between them have comparable cleansing efficacy. This post hoc analysis explored the attainment of high-quality bowel cleansing success at the patient and segment population levels.

High-quality cleansing was attained more frequently in patients receiving NER1006 versus 2LPEG or OSS. The difference was small, but statistically significant, and present also in the predefined original analysis population, mFAS. Notably, in this population in the two independent randomized controlled trials MORA and NOCT, NER1006 was the only treatment to achieve a $45 \%$ or higher rate of high-quality cleansed segments and it did so reproducibly in two trials. 
- Table 1 Comparative attainment in mFAS2 of PDR and ADR per treatment at a combined trial level.

\begin{tabular}{|c|c|c|c|c|c|c|c|c|c|c|}
\hline $\begin{array}{l}\text { Min number of neoplasia } \\
\text { per patient }\end{array}$ & 1 & 2 & 3 & 4 & 5 & 6 & 7 & 8 & 9 & 10 \\
\hline \multicolumn{11}{|l|}{ Patients with polyps ${ }^{1}$} \\
\hline NER1006 patients, $\mathrm{n}$ & $243 / 517$ & $128 / 517$ & $84 / 517$ & $52 / 517$ & $30 / 517$ & $24 / 517$ & $22 / 517$ & $18 / 517$ & $12 / 517$ & $9 / 517$ \\
\hline NER1006 patients, \% (PDR) & 47.0 & 24.8 & 16.3 & 10.1 & 5.8 & 4.6 & 4.3 & 3.5 & 2.3 & 1.7 \\
\hline 2LPEG/OSS patients, $\mathrm{n}$ & $253 / 520$ & $133 / 520$ & $79 / 520$ & $43 / 520$ & $28 / 520$ & $20 / 520$ & $10 / 520$ & $5 / 520$ & $4 / 520$ & $3 / 520$ \\
\hline 2LPEG/OSS patients, \% (PDR) & 48.7 & 25.6 & 15.2 & 8.3 & 5.4 & 3.9 & 1.9 & 1.0 & 0.8 & 0.6 \\
\hline Difference, \% & -1.65 & -0.82 & 1.06 & 1.79 & 0.42 & 0.80 & 2.33 & 2.52 & 1.55 & 1.16 \\
\hline \multicolumn{11}{|l|}{ Patients with adenomas ${ }^{2}$} \\
\hline NER1006 patients, $\mathrm{n}$ & $163 / 508$ & $81 / 508$ & $45 / 508$ & $26 / 508$ & $15 / 508$ & $14 / 508$ & $10 / 508$ & $8 / 508$ & $5 / 508$ & $3 / 508$ \\
\hline NER1006 patients, \% (ADR) & 32.1 & 15.9 & 8.9 & 5.1 & 3.0 & 2.8 & 2.0 & 1.6 & 1.0 & 0.6 \\
\hline 2LPEG/OSS patients, $n$ & $168 / 508$ & $70 / 508$ & $39 / 508$ & $22 / 508$ & $12 / 508$ & $7 / 508$ & $4 / 508$ & $2 / 508$ & $2 / 508$ & $2 / 508$ \\
\hline 2LPEG/OSS patients, \% (ADR) & 33.1 & 13.8 & 7.7 & 4.3 & 2.4 & 1.4 & 0.8 & 0.4 & 0.4 & 0.4 \\
\hline Difference, \% & -0.98 & 2.17 & 1.18 & 0.79 & 0.59 & 1.38 & 1.18 & 1.18 & 0.59 & 0.20 \\
\hline \multicolumn{11}{|c|}{$\begin{array}{l}\text { PDR, polyp detection rate; ADR, adenoma detection rate; } 2 \text { LPEG, } 2 \text { L polyethylene glycol with ascorbate; OSS, oral sulfate solution. } \\
1 \text { Patients with at least one polyp. } \\
2 \text { Patients with at least one adenoma. }\end{array}$} \\
\hline
\end{tabular}

Because the mFAS population included a number of patients who for conservative reasons were imputed as overall cleansing failures, the overall colon cleansing efficacy was also assessed in mFAS2. The segmental scores distribution analysis suggests that NER1006 achieves its improved cleansing mainly by delivering more stool-free segments and fewer segments with removable stool.

It is intriguing that subtle but statistically significant and consistent improvements in cleansing quality can make a practical difference in lesion detection. Positive trends, numerical only but conceptually compatible, were identified towards detection of more patients with multiple neoplasia and also more neoplasia per patient in those patients who had more than just one. This is particularly interesting since these analyses were performed in relatively small sample sizes that had been optimized for cleansing quality assessment. A larger study should ideally randomize patients for adenoma presence (or not), the number of adenomas per patient, and finally the size of each adenoma; since that is not possible these analyses were justified for that reason alone.

Considering the increasing evidence base for small and diminutive adenomas on the one hand, and adenoma multiplicity on the other, as risk factors for CRC, our results indicate that some adenomas may have been missed with current treatments. It is plausible that patients with one to two adenomas had them detected only because of their large size, i. e. they were detectable even in a stool-containing bowel.

User-friendliness was not a focus for the current analysis; however, on the day before colonoscopy, NER1006 requires only a total fluid intake of $2 \mathrm{~L}$, less than for either $2 \mathrm{LPEG}$ or OSS, and NER1006 may also present an increased patient benefit, particularly compared with OSS, by permitting both break- fast and lunch. Apart from convenience, avoidance of unnecessary fasting periods significantly improves the nutritional status of elderly and hospitalized patients [33].

These post hoc analyses have several strengths. Based on two randomized Phase III clinical trials with near-identical study design, these analyses evaluated comparative cleansing efficacy in highly comparable and strictly assessed data sets; for instance, all patients in the mFAS2 had documented segmental cleansing scores and we only analyzed comparable dosing regimens for each treatment. This enabled a true comparative efficacy analysis between bowel preparations when used as per the label.

The limitations of these analyses include their post hoc nature as well as the potential for inter-patient variability for having any neoplasia at all, and if so, then how many and of what size. Adenoma size was not monitored in the analyzed trials. Statistical limitations include lack of adjustments for possible repeat effects in trends or type 1 errors (false positives) with some very small sample sizes. Trend analyses are not presented, but were considered. Recommended adjusted tests such as the two-sided Kolmogorov-Smirnov test either failed to capture the possibility of missed neoplasia in the comparator group (falsely assuming that all patients with, for example, two adenomas had none more to be detected) or could be accused for analysis back-fitting to results if for instance the max cumulative adenomas detection limit of 2LPEG/OSS was set to only $85 \%$ versus $100 \%$ for NER 1006 (as demonstrated by actual outcomes). A potential limitation of this analysis was also the lack of comparison with $4 \mathrm{~L}$ PEG, which was never tested in the MORA and NOCT trials. Two recent real-world studies, however, suggest that all three treatments in the current analysis deliver 
higher mean cleansing scores than 4L PEG on the Boston Bowel Preparation Scale [34, 35].

\section{Conclusion}

In conclusion, in patients with Harefield Cleansing Scale scores assessed by treatment-blinded central readers, NER1006 (PLENVU) delivered more high-quality cleansed segments than 2LPEG or OSS. It is currently not possible to prospectively randomize patients to have a given number or size of neoplasia. Considering recently published studies on the positive effects of treatment-independent high-quality cleansing on ADR and MAP, our post hoc analysis of polyp and adenoma multiplicity detection supports the premise that the improved high-quality cleansing with NER1006 may facilitate the detection of multiple neoplasia in patients. NER1006 could thereby help improve patient protection against CRC.

\section{Acknowledgements}

Medical writing support for the preparation of this manuscript was kindly provided by Lucy Clayton at Norgine.

\section{Competing interests}

Michael Epstein: was an investigator in the NOCT study and has acted as a safety advisor for Aspire Bariatrics, a consultant for Zx Pharma and IM HealthScience, and a speaker for Daichi Sankyo and Pfizer; Robert Benamouzig: was an investigator in the MORA study, has attended advisory board for Medtronics, Fujy, and Alfasigma, and acted as speaker for Mayoli; Juha Halonen, Employee of Norgine; Raf Bisschops: was an investigator in the MORA study and has received honoraria from Norgine for speaking and advisory board attendance.

\section{References}

[1] Brenner H, Stock C, Hoffmeister M. Effect of screening sigmoidoscopy and screening colonoscopy on colorectal cancer incidence and mortality: systematic review and meta-analysis of randomised controlled trials and observational studies. BMJ 2014; 348: g2467

[2] Lin OS, Kozarek RA, Cha JM. Impact of sigmoidoscopy and colonoscopy on colorectal cancer incidence and mortality: an evidence-based review of published prospective and retrospective studies. Intest Res 2014; 12: 268-274

[3] Nishihara R, Wu K, Lochhead P et al. Long-term colorectal-cancer incidence and mortality after lower endoscopy. N Engl J Med 2013; 369; 1095-1105

[4] Corley DA, Jensen CD, Marks AR et al. Adenoma detection rate and risk of colorectal cancer and death. N Engl J Med 2014; 370: 12981306

[5] Kaminski MF, Wieszczy P, Rupinski M et al. Increased rate of adenoma detection associates with reduced risk of colorectal cancer and death. Gastroenterology 2017; 153: 98-105

[6] Amano T, Nishida T, Shimakoshi $\mathrm{H}$ et al. Number of polyps detected is a useful indicator of quality of clinical colonoscopy. Endosc Int Open 2018; 6: E878-E884

[7] Gessl I, Waldmann E, Penz D et al. Evaluation of adenomas per colonoscopy and adenomas per positive participant as new quality parameters in screening colonoscopy. Gastrointest Endosc 2019; 89: 496-502

[8] Oh CH, Lee CK, Kim J-W et al. Suboptimal bowel preparation significantly impairs colonoscopic detection of non-polypoid colorectal neoplasms. Dig Dis Sci 2015; 60: 2294-2303

[9] Clark BT, Protiva P, Nagar A et al. Quantification of adequate bowel preparation for screening or surveillance colonoscopy in men. Gastroenterology 2016; 150: 396-405; quiz e14-15

[10] Clark BT, Laine L. High-quality bowel preparation is required for detection of sessile serrated polyps. Clin Gastroenterol Hepatol 2016; 14: 1155-1162

[11] Pontone S, Hassan C, Maselli R et al. Multiple zonal and multi-zone adenoma detection rates according to quality of cleansing during colonoscopy. United Eur Gastroenterol J 2016; 4: 778-783

[12] Hassan C, Bisschops R, Heimanson Z et al. Tu1074 Impact of cleansing quality using the Harefield Cleansing Scale and polyp and adenoma detection rates: a post hoc analysis of three phase 3 randomized trials. Gastrointest Endosc 2018; 87: ab521-ab522

[13] Guo R, Wang Y-J, Liu M et al. The effect of quality of segmental bowel preparation on adenoma detection rate. BMC Gastroenterol 2019; 19: 119

[14] Laish I, Sergeev I, Stein A et al. Risk of metachronous advanced lesions after resection of diminutive and small non-advanced adenomas. Clin Res Hepatol Gastroenterol 2019; 43: 201-207

[15] Kim NH, Jung YS, Lee MY et al. Risk of developing metachronous advanced colorectal neoplasia after polypectomy in patients with multiple diminutive or small adenomas. Am J Gastroenterol 2019; 114: 1657-1664

[16] Vleugels JLA, Hassan C, Senore C et al. Diminutive polyps with advanced histologic features do not increase risk for metachronous advanced colon neoplasia. Gastroenterology 2019; 156: 623-634.e3

[17] Anderson JC, Rex DK, Robinson C et al. Association of small versus diminutive adenomas and the risk for metachronous advanced adenomas: data from the New Hampshire Colonoscopy Registry. Gastrointest Endosc 2019; 90: 495-501

[18] Hartstein JD, Vemulapalli KC, Rex DK. The predictive value of small versus diminutive adenomas for subsequent advanced neoplasia. Gastrointest Endosc 2019; 91: 614-621

[19] Løberg M, Kalager M, Holme Ø et al. Long-term colorectal-cancer mortality after adenoma removal. N Engl J Med 2014; 371: 799-807

[20] Bonnington SN, Sharp L, Rutter MD. Post-polypectomy surveillance in the English bowel cancer screening programme: results of first surveillance. Endoscopy 2019; 51: S116.OP335

[21] Bonnington SN, Sharp L, Rutter MD. Post-polypectomy surveillance in the English bowel cancer screening programme: multivariate logistic regression of factors influencing advanced adenoma detection at first surveillance. Endoscopy 2019; 51: S153.ePP79

[22] Lieberman D, Sullivan BA, Hauser ER et al. Baseline colonoscopy findings associated with 10-year outcomes in a screening cohort undergoing colonoscopy surveillance. Gastroenterology 2020; 158: 862874.e8

[23] Meester RG, Doubeni CA, Lansdorp-Vogelaar I et al. Variation in adenoma detection rate and the lifetime benefits and cost of colorectal cancer screening: a microsimulation model. JAMA 2015; 313: 23492358

[24] Zimmermann-Fraedrich K, Sehner S, Rex DK et al. Right-sided location not associated with missed colorectal adenomas in an individual-level reanalysis of tandem colonoscopy studies. Gastroenterology 2019; 157: 660-671

[25] Halphen M, Heresbach D, Gruss H-J et al. Validation of the Harefield Cleansing Scale: a tool for the evaluation of bowel cleansing quality in both research and clinical practice. Gastrointest Endosc 2013; 78: $121-131$ 
[26] IQVIA MIDAS World Review Pack 2019. Market: Worldwide market of bowel cleansing products only (excl. enemas). Data supplied by IQVIA and analysed by Norgine

[27] DeMicco MP, Clayton LB, Pilot J et al. Novel 1 L polyethylene glycolbased bowel preparation NER1006 for overall and right-sided colon cleansing: a randomized controlled phase 3 trial versus trisulfate. Gastrointest Endosc 2018; 87: 677-687.e3

[28] Bisschops R, Manning J, Clayton LB et al. Colon cleansing efficacy and safety with $1 \mathrm{~L}$ NER 1006 versus $2 \mathrm{~L}$ polyethylene glycol + ascorbate: a randomized phase 3 trial. Endoscopy 2019; 51: 60-72

[29] Schreiber S, Baumgart DC, Drenth JPH et al. Colon cleansing efficacy and safety with $1 \mathrm{~L}$ NER1006 versus sodium picosulfate with magnesium citrate: a randomized phase 3 trial. Endoscopy 2019; 51: 73-84

[30] Kane SV, Ananthakrishnan AN. Toward reducing bias in clinical trials: central readers for endoscopic endpoints. Gastrointest Endosc 2016; 83: $198-200$
[31] Kim B, Lee SD, Han KS et al. Comparative evaluation of the efficacy of polyethylene glycol with ascorbic acid and an oral sulfate solution in a split method for bowel preparation: a randomized multicenter phase III clinical trial. Dis Colon Rectum 2017; 60: 426-432

[32] Lee HH, Lim CH, Kim JS et al. Comparison between an oral sulfate solution and a $2 \mathrm{I}$ of polyethylene glycol+ascorbic acid as a split dose bowel preparation for colonoscopy. J Clin Gastroenterol 2019; 53: e431-e437

[33] Nunes G, Barata AT, Santos CA et al. Nutritional deficiency during colonoscopy preparation: the forgotten iatrogeny. Rev Esp Enferm Dig 2018; 110: 285-291

[34] Gu P, Lew D, Oh S] et al. Comparing the real-world effectiveness of competing colonoscopy preparations: results of a prospective trial. Am J Gastroenterol 2019; 114: 305-314

[35] Maida M, Morreale G, Sinagra E et al. Effectiveness and tolerability of very low-volume preparation for colonoscopy: a prospective multicenter observational study. Endoscopy 2019; 51: S70 